\title{
Diseño de libros para dispositivos de tinta electrónica
}

\author{
Por Pablo Lara-Navarra, Begoña Gros-Salvat y Magí Almirall
}

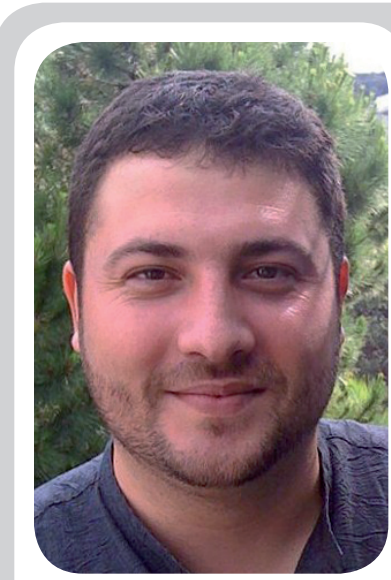

Pablo Lara-Navarra es director de Innovación de la Universitat Oberta de Catalunya y director académico del Master en Tecnologías Accesibles para la Sociedad de la Información. Es miembro del Grupo ThinkEPI y de los grupos de investigación Tecnologías de la información, universidad y sociedad red (Ituns) y Entornos y materiales para el aprendizaje (EMA).

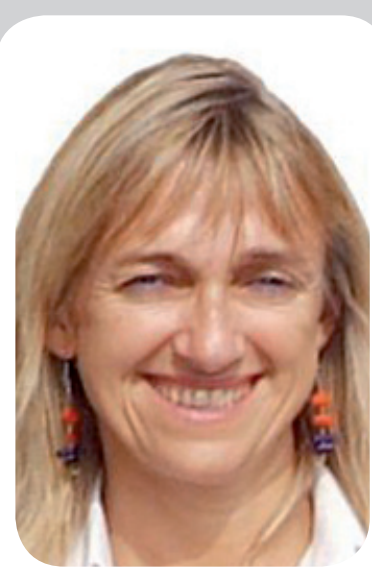

Begoña Gros-Salvat es vicerrectora de Innovación de la Universitat Oberta de Catalunya. Especialista en el uso de las tecnologías de la información y la comunicación para el aprendizaje. Dirige el grupo de Investigación Entornos y materiales para el aprendizaje (EMA) de la Univ. de Barcelona.

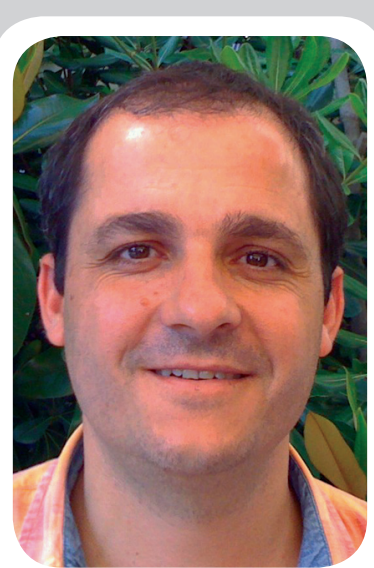

Magí Almirall és director de Tecnología Educativa de la UOC. Basa sus proyectos en estándares, usabilidad, accesibilidad y software libre. Su equipo recibió el premio a la Innovación digital del congreso IGC 2006 y el premio a la mejor solución de e-learning para móviles IMS Gold 2008.
Resumen: Las tecnologías multiplataforma y multicanal permiten la movilidad de la información, cosa que tiene una incidencia directa sobre la evolución del libro electrónico. El cambio viene dado por la aparición de una nueva generación de dispositivos de tinta electrónica, así como por los propios modelos de gestión de la información. En el presente trabajo se revisa el concepto de libro electrónico y se presentan las características básicas de los dispositivos que se pueden encontrar en el mercado, para a continuación exponer los elementos necesarios para la ejecución de proyectos de producción de obras para dispositivos de tinta electrónica.

Palabras clave: Movilidad de información, Libro electrónico, Multiplataforma, Dispositivos de tinta electrónica

\section{Title: Design of e-books to be used in e-ink devices}

Abstract: The multi-channel and multi-platform technologies allow the mobility of information, which has a direct impact on the evolution of the electronic book. The change is dictated by the emergence of a new generation of electronic paper devices, as well as their own models of information management. In the present paper we review the electronic book concept and present the basic characteristics of the devices that can be found on the market; then we describe the elements needed to carry out projects that produce works designed for electronic ink devices.

Keywords: Information mobility, Electronic books, eBook, Multiplatform, Electronic ink devices

Lara-Navarra, Pablo; Gros-Salvat, Begoña; Almirall, Magi. "Diseño de libros para dispositivos de tinta electrónica”. En: El profesional de la información, 2008, julio-agosto, v. 17, n. 4, pp. 390-395.

DOI: 10.3145/epi.2008.jul.03

\section{Introducción}

La idea de que la tecnología evoluciona con gran rapidez es un pensamiento compartido por todos los profesionales que trabajan en el mundo electrónico, y esta sensación se encuentra sintetizada en la frase inglesa "technology is moving fast". Este pensamiento de celeridad y cambio actualmente viene acentuado por otra variable: la movilidad. La introducción de este concepto altera sustancialmente el escenario de las tecnologías de la información y, en consecuencia, afecta a los sistemas de gestión de información. En este trabajo se tratará de la evolución de los modelos de producción de libros electrónicos y del mercado de dispositivos móviles para su lectura.

¿En qué afecta la introducción del factor movilidad a los procesos de gestión de información electrónica? Adoptando sintéticamente como hilo argumental la historia de la Web, recordaremos que las primeras páginas eran estáticas (html), evolucionando luego a un modelo de contenidos dinámicos en la que la información se encuentra estructurada (xml). A partir de aquí surgen diferentes tecnologías para la cooperación (web 2.0), descentralización (escritorios virtuales), y sindicación (rss). 
Centraremos la atención en la sindicación de contenidos, donde la rss (really simple syndication) se puede considerar como el primer sistema que impulsa y estabiliza el desarrollo de tecnologías que modifican los modelos de gestión de información electrónica de forma sustancial: el usuario no busca el contenido ( $p u l l$ ), sino que es el contenido el que encuentra al usuario (push). Esta transformación permite asentar el término "información móvil" para referirse a contenidos que pueden saltar de un sistema a otro en base a unos principios, que son:

- Multiplataforma. La información electrónica debe ser adaptable a las plataformas más extendidas en cada uno de sus formatos para alcanzar al máximo número de usuarios.

- Multicanal. Los usuarios podrán tener acceso a la información a través de distintos canales para adaptarse a los distintos perfiles de interacción, acceso y conocimiento.

A día de hoy, la información puede ser estandarizada, estructurada y sindicada, y además pueden añadirse a la misma capas multiplataforma y multicanal, para que se pueda empaquetar y ser leída a través de diferentes canales (televisión, radio, web...) sin depender de la plataforma (windows, linux, mac, windows mobile, android...). Es posible generar libros electrónicos con diferentes formatos de salida (papel, web, mp3) que pueden visualizarse en cualquier dispositivo, como por ejemplo los libros de tinta electrónica.

\section{"La movilidad es uno de los factores básicos a considerar en la producción de información electrónica a principios del siglo XXI"'}

En resumen, la evolución de los elementos anteriormente descritos ha obligado a desarrollar nuevos modelos de gestión de información y a reformular el concepto de libro electrónico.

Ante el atractivo de los nuevos mercados generados por las tecnologías móviles, diferentes ámbitos profesionales buscan tener un papel protagonista, como es el caso de las ingenierías informáticas, pero también otros campos de conocimiento pueden y deben adoptar un papel relevante en los mismos. Un actor obligatorio es el profesional de las ciencias de la información y documentación. Su papel viene determinado por la necesidad de que haya un interlocutor entre el usuario y el dispositivo, un gestor de información, alguien con capacidad de liderar proyectos de libros electrónicos para dispositivos de tinta electrónica, mejorando la in- teracción con el usuario en las diferentes necesidades y en cualquier ambiente de lectura.

\section{Evolución del libro electrónico}

Las formas que ha ido adoptando la publicación electrónica pueden haber impedido que se tengan conceptos claros universalmente aceptados. Hace unas décadas la idea era fácil de entender, pues se concretaba en informatizar los libros físicos. Posteriormente se dispuso de herramientas que permiten que los procesos de publicación sean totalmente electrónicos: creación de la obra, corrección de textos, maquetación, corrección de pruebas, impresión directa..., hasta llegar a la fase final de producción en la que, como se ha dicho, se puede elegir entre diferentes formatos de salida: papel, web, fichero de voz, etc.

Así pues, en la actualidad el proceso de gestión del libro siempre es electrónico, sea cual sea el formato de salida.

La definición de libro de la RAE continúa siendo válida para el libro electrónico: "Obra científica, literaria o de cualquier otra índole con extensión suficiente para formar volumen, que puede aparecer impresa o en otro soporte". Sin embargo, aunque el concepto aún sea vigente, los libros-e han ido experimentando cambios técnicos importantes que cada vez les han hecho más atractivos, hasta llegar al momento actual en que están a punto de convertirse en un producto de masas.

En un primer estadio, cuando comenzó a generalizarse el uso del ordenador, el libro electrónico estuvo limitado por la baja calidad de la lectura en pantalla, la escasa interoperabilidad de los diferentes periféricos del ordenador y su pobre comportamiento ante la interacción humana. El ratón, el aumento de la velocidad de barrido de las pantallas o las tarjetas gráficas mejoraron sustancialmente la ergonomía y la capacidad de almacenamiento. A partir de aquí los esfuerzos se desplazaron hacia hallar un software que pudiera replicar el imaginario del libro físico, llegándose a imitar las acciones que se hacen con un libro de papel, como por ejemplo pasar páginas. Por consiguiente, se podría establecer que la evolución del libro electrónico ha dependido de los avances del campo de conocimiento Interacción Persona Ordenador (IPO).

La mayoría de especialistas marcan cuatro hitos en la historia del libro electrónico (Millán, José-Antonio; Codina, Lluís):

- 1971. Primeros libros digitales a cargo de Michael Hart, de la Universidad de Illinois, fundador del Proyecto Gutenberg, una biblioteca gratuita de libros digitales con más de 22.000 títulos entre los que se encuentra un gran número de obras clásicas. Gracias a los modernos medios de escaneo y al número de vo- 
luntarios Hart espera ofrecer 1 millón de libros en 100 idiomas en 2015.

- 1981. Publicación del Random House's Electronic Dictionary, probablemente el primer "libro electrónico" disponible comercialmente en el mundo.

- 1998. Se realiza la primera feria del libro electrónico en Gaithersburg, Maryland, Estados Unidos. Allí se presentó la normativa "Libro electrónico abierto" (Open eBook Publication Structure).

- 2001. Puesta en venta del libro electrónico Riding the Bullet, de Stephen King, que logró en 24 horas la cifra de 400.000 descargas de su novela.

Existen ya muchos otros proyectos de libros electrónicos, siendo Google Books y el dispositivo Kindle, de Amazon, los más importantes y mediáticos en estos últimos años.

En resumen, la actividad relacionada con el libro electrónico a día de hoy tiene dos grandes vertientes: la gestión de contenidos para producir libros en formato electrónico, y el diseño de dispositivos que ayuden a su integración en la vida cotidiana.

Dentro de la segunda área de actividad, este trabajo centra su estudio en los dispositivos de lectura que usan tinta electrónica. Wikipedia define tinta electrónica o papel electrónico como "una tecnología que permite crear pantallas planas, tan delgadas como un papel, y con una flexibilidad que se puedan enrollar. Estas pantallas por el momento sólo pueden representar información estática y en blanco y negro"

De forma sintetizada, Ramón Rey en su entrada Tinta electrónica, indica que la clave de la tecnología se encuentra en la capa intermedia, donde millones de cápsulas blancas por un lado y negras por el otro flotan en un polímero en estado de gel que les permite moverse libremente. Esta capa está entre otras dos formadas por microelectrodos que transmiten impulsos eléctricos a través del polímero de manera que las cápsulas se orientan en la pantalla y así se representan textos y gráficos.

Existen dos líneas de innovación tecnológica para el diseño de dispositivos, ambas en competición, que pueden tener su propio mercado, e incluso puede suceder que sus características permitan darles usos diferentes. Son desarrolladas por Xerox y E-Ink:

- La tecnología de Xerox emplea esferas con carga positiva y negativa, la parte positiva es de color negro y la negativa de color blanco, y según el impulso eléctrico se mostrará en la superficie un color u otro.

- E-Ink usa partículas de titanio de colores blanco y negro. En este caso se pueden visualizar las partículas negras, las blancas o sólo la mitad de ellas.

Los sistemas citados no sólo son empleados en la producción de lectores de libro electrónicos. También encuentran su uso en carteles electrónicos para la publicidad en escaparates, pues permiten ir refrescando la información sin necesidad de grandes pantallas y con un consumo energético muy bajo, así como se han hecho señales de tráfico. Si embargo el mercado más atractivo son los libros.

Tras este breve recorrido por el concepto, y una vez centrados en los dispositivos de tinta electrónica, el primer paso será verificar qué productos existen en el mercado y qué características tienen.

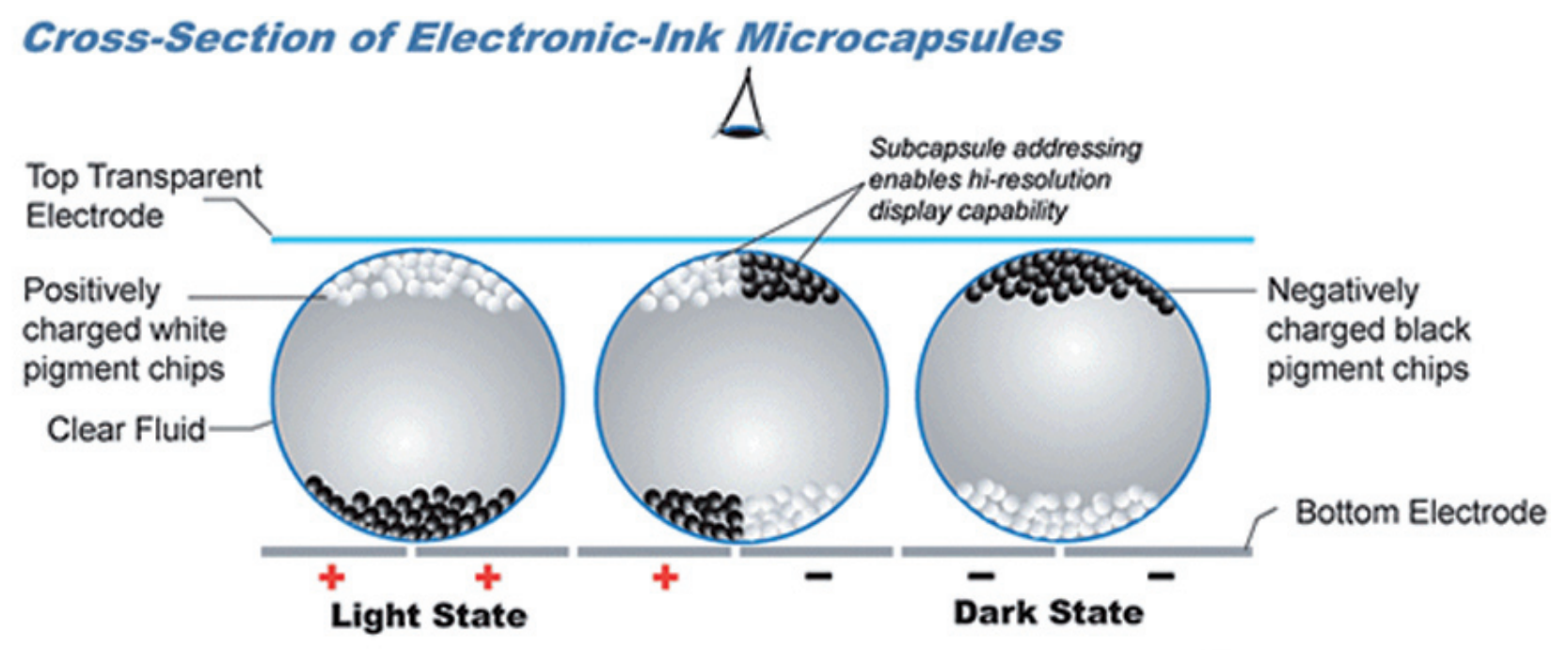

NOTE: Copyright E Ink Corporation, 2002. Image not drawn to scale - for illustration purposes only. 
Principales dispositivos de tinta electrónica y sus características

\begin{tabular}{|c|c|}
\hline Papyre 6.1 & Producido en Granada por la empresa española Facthor con arquitectura Linux \\
\hline & $\begin{array}{l}\text { - Tamaño de pantalla: } 6 " \\
\text { - Resolución: } 600 \times 800 \\
\text { - Formatos de texto soportados: PDF, HTML, TXT... } \\
\text { - Formato de audio: MP3 }\end{array}$ \\
\hline \multirow[t]{2}{*}{ Sony Reader } & $\begin{array}{l}\text { Distribuido en los EUA, con una medida más pequeña que el Sony Librie. Este libro } \\
\text { trabaja igualmente en PDF y formato propio, del cual facilitan un conversor. }\end{array}$ \\
\hline & $\begin{array}{l}\text { - Tamaño de pantalla: } 6 " \\
\text { - Resolución: } 600 \times 800 \\
\text { - Formatos de texto soportados: BBeB Book (BroadBand eBook o LRS), Adobe PDF, } \\
\text { TXT, RTF y DOC (en este último caso se necesita tener instalado Microsoft Word } \\
\text { en el PC para realizar la conversión) } \\
\text { - Formatos de audio: MP3 y AAC } \\
\text { - Formatos de imagen: JPEG, GIF, PNG y BMP }\end{array}$ \\
\hline \multirow[t]{2}{*}{ iRex Iliade } & $\begin{array}{l}\text { Distribuido por iRex (Philips) en Europa, este dispositivo cuenta con pantalla táctil } \\
\text { y tiene la posibilidad de marcar notas con un lápiz sobre los documentos. Incluye } \\
\text { conexión WiFi. }\end{array}$ \\
\hline & $\begin{array}{l}\text { - Tamaño de pantalla: } 8,1^{\prime \prime} \\
\text { - Resolución: } 768 \times 1024 \\
\text { - Formatos de texto soportados: PDF, HTML, TXT y PRC (el de la tienda online Mo- } \\
\text { bipocket) } \\
\text { - Formatos de imagen: JPEG, PNG y BMP }\end{array}$ \\
\hline \multirow[t]{2}{*}{ Amazon Kindle } & $\begin{array}{l}\text { Fabricado por Amazon actualmente sólo se distribuye en EUA. Este producto se } \\
\text { encuentra unido a la tienda virtual, a formatos propietarios y a redes de datos pre- } \\
\text { sentes únicamente en el mercado americano. }\end{array}$ \\
\hline & $\begin{array}{l}\text { - Tamaño de pantalla: 6" } \\
\text { - Resolución: } 600 \times 800 \\
\text { - Formatos de texto soportados: TXT, AZW, PRC, MOBI } \\
\text { - Formato de audio: MP3 }\end{array}$ \\
\hline \multirow[t]{2}{*}{ Cybook Gen3 } & Fabricado por Bookeen en Francia \\
\hline & $\begin{array}{l}\text { - Tamaño de pantalla: 6" } \\
\text { - Resolución: } 600 \times 800 \\
\text { - Formatos de texto soportados: PDF, MOBI, PRC, PalmDOC, HTML, TXT } \\
\text { - Formato de audio: MP3 } \\
\text { - Formatos de imagen: JPEG, GIF, PNG }\end{array}$ \\
\hline \multirow[t]{2}{*}{ STAReBOOK STK-101 } & Distribuido desde China por eREAD Holding Co \\
\hline & $\begin{array}{l}\text { - Tamaño de pantalla: } 6 " \\
\text { - Resolución: } 600 \times 800 \\
\text { - Formatos de texto soportados: TXT, PDF, DOC... } \\
\text { - Formato de audio: MP3 } \\
\text { - Formato de imagen: JPG }\end{array}$ \\
\hline
\end{tabular}

A partir de este punto y tras comprobar que existen dispositivos para llevar a cabo proyectos de tinta electrónica, se puede iniciar el proceso de realización de proyectos.

\section{Etapas básicas de un proyecto de libro de tinta electrónica}

Se propone un método que ya se ha aplicado experimentalmente, adaptación del "desarrollo en espiral", de Barry Boehm, ampliamente probado. Consiste en realizar cada parte del proyecto de forma parcial y probarla con los usuarios hasta conseguir la versión final del conjunto. El proyecto se divide en pequeños objetivos y a cada uno se le aplican de forma cíclica las típicas fases de definición, desarrollo, ejecución y evaluación. Al acabar cada ciclo se inicia otro, incorporando las conclusiones de la evaluación y nuevos objetivos.

Así, adaptando las 4 fases del ciclo espiral, proponemos: 


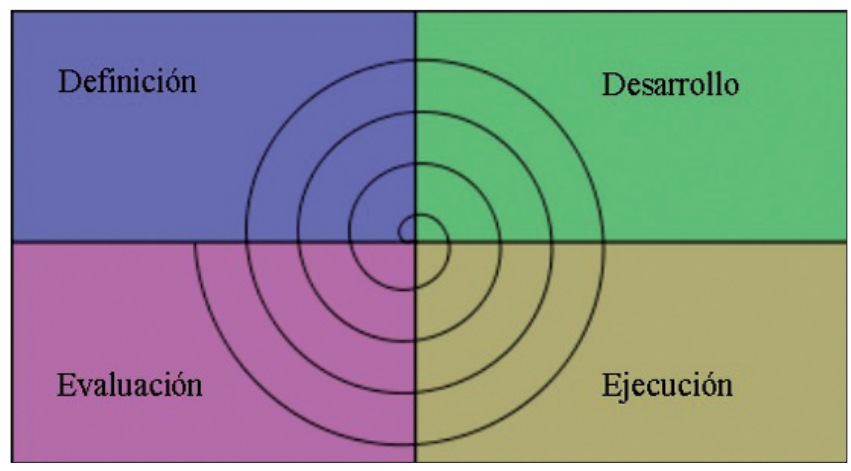

Gráfico 2: Modelo de desarrollo espiral

\section{Fase 1. Definición y elección del dispositivo}

Es una fase clave, puesto que el dispositivo marcará gran parte del resultado. Tal y como se ha visto anteriormente, las opciones son múltiples y sus diferencias han de determinar nuestra decisión.

Si se quiere iniciar una prueba y crecer de forma espiral es mejor buscar la simplicidad. Los dispositivos ofrecen opciones interesantes que deben ajustarse a los objetivos que fijemos. Destacamos especialmente:

- opción wifi, indispensable si se quiere la actualización de los libros de forma continua;

- lápiz magnético, muy valorado en entornos de aprendizaje donde el estudiante y el profesor pueden compartir anotaciones y comentarios;

- tamaño de la pantalla, especialmente relevante en formatos PDF ya que el contenido de las páginas es fijo y por tanto podríamos estar obligando al lector a usar constantemente el zoom.

Existen otros aspectos de interés al elegir el dispositivo, como el sistema operativo base, ya que algunos trabajan con licencias de software libre y por tanto nos dan la posibilidad de modificarlo; o los formatos aceptados por el dispositivo, típicamente PDF y HTML, acompañados en algunos casos de formatos propietarios como el BBeB de Sony.

\section{Fase 2. Desarrollo y preparación de los contenidos}

Incluye la preparación del material bibliográfico (libros, revistas, folletos...) a incorporar en el dispositivo así como el envío a los usuarios. Los formatos empleados marcarán la facilidad de uso, y a su vez su aceptación por parte del usuario final. No se trata de insertar un PDF ya existente de tamaño A4 en un dispositivo de 6 " u 8" de pantalla. Es necesario reeditar el contenido adaptándolo a las medidas pero especialmente ajustándolo a las funcionalidades del dispositivo para así sacar el mayor partido del salto al libro electrónico: aumento del tamaño de la letra, búsquedas, notas personales sobre el texto son valiosas funcionalidades que debemos asegurar que se pueden usar con los contenidos que hemos insertado. Por ejemplo, si introducimos contenido textual en formato imagen vamos a perder la función de búsqueda, y si optamos por el formato PDF perderemos la capacidad de repaginar el libro al cambiar el tamaño de la letra.

\section{"No se trata de insertar un pdf ya existente de tamaño A4 en un dispositivo de 6 pulgadas de pantalla"}

Así, el esfuerzo en este segundo paso puede resultar importante, y hasta puede representar una reedición o un reenfoque del contenido. Por este motivo la automatización de la creación de los formatos a partir de XML es fundamental para el éxito del proyecto. Desde XML se pueden obtener formatos de contenidos a medida, y fabricar estructuras en PDF adaptando el contenido al dispositivo. Para conseguirlo se usan las especificaciones XSL (extensible stylesheet language) del W3C (WWW Consortium) de presentación de la información, denominadas FO (formatting objects), pasando el contenido XML a PDF con FOP (formatting object processor). Esta propuesta de automatización facilita el proyecto pero no es indispensable ya que se puede también adaptar el contenido manualmente y crear los pdf o html necesarios a partir de editores de texto comunes como Word u OpenOffice.

\section{Fase 3. Ejecución, seguimiento y formación}

Dado el carácter novedoso del libro-e es necesario realizar un proyecto piloto con usuarios, sobre los cuales se hacen acciones de formación y seguimiento. El paquete de evaluación incluye aspectos de mejora de la usabilidad que deberán disminuir la curva de aprendizaje y minimizar el esfuerzo en formación.

Es recomendable usar una web para gestionar esta parte del proyecto. Los usuarios esperan instrucciones claras e inmediatas y en un espacio virtual podemos explotar la información textual con vídeos y fotos que faciliten la comprensión. Una nueva tecnología tiene nuevos conceptos que pueden chocar completamente con la lógica del usuario. Por ejemplo, en un dispositivo de tinta electrónica al acabarse la batería no desaparece la información de la pantalla sino que queda la pantalla inmóvil, ya que sin batería no puede cambiar de página. Delante de una pantalla de texto inmóvil el usuario tiende a pensar en una avería, no que se ha quedado sin 


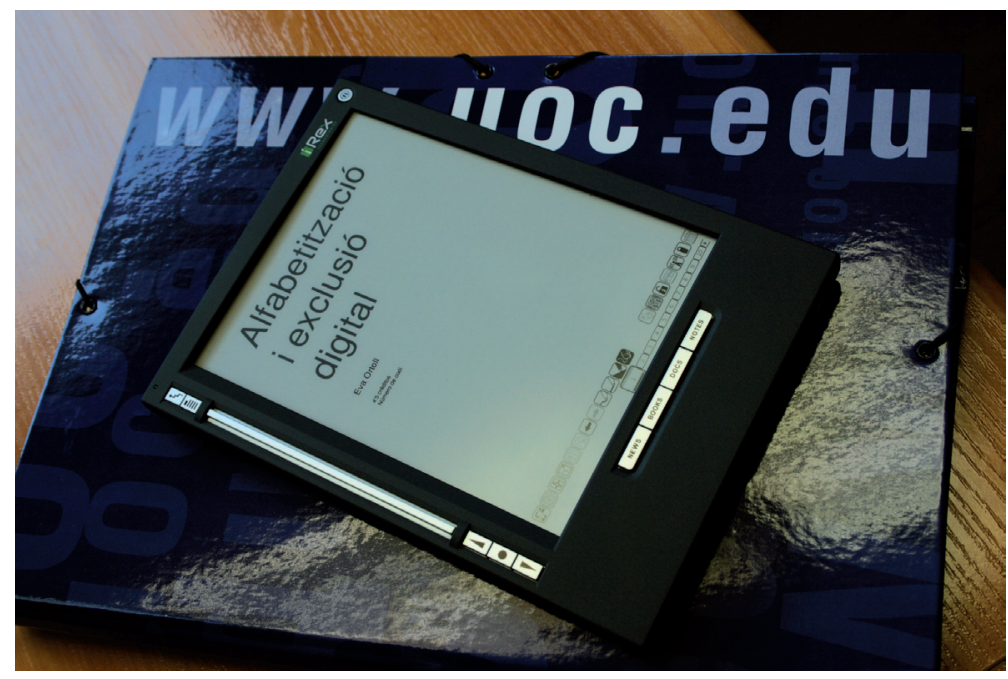

Gráfico 3: Dispositivo de tinta electrónica

batería. Este ejemplo concreto provocó la devolución errónea de dos dispositivos en una prueba piloto de 10 usuarios. Así, proponemos la edición de vídeos de uso como información básica.

Véase como ejemplo el vídeo creado por el equipo de la primera prueba piloto de tinta electrónica en la UOC:

http://www.youtube.com/watch? $v=O O n V z 24 c T 3 M$

El seguimiento y la evaluación del proyecto han de ser coherentes y coordinados. Así, en la fase de evaluación analizaremos los resultados del trabajo de seguimiento que proponemos usando la "observación participante" (Beyer, Hugh; Holtzblatt, Karen) que se detalla en el siguiente apartado. Tener en cuenta el punto de vista de los usuarios, aparte de los aspectos técnicos y de forma de leer el dispositivo, permitirá obtener propuestas de mejora que reduzcan significativamente la curva de aprendizaje, y que harían innecesaria una acción formativa en caso de llevarse a cabo una implementación global del dispositivo.

\section{Fase 4. Evaluación y observación participante}

Dado que en una prueba piloto el volumen de usuarios es bajo, se realiza una evaluación cualitativa basada, como se ha mencionado, en la observación participante: el evaluador y sus herramientas se introducen en el sistema intentando acercarse al máximo al usuario y a su entorno real. Así, se sugieren dos métodos concretos: por una parte la observación del entorno y por otra el cuaderno de campo.

Para la observación de entorno es necesario conseguir que un usuario acepte tener a su lado al observador durante el tiempo y en el lugar donde use el dispositivo; el observador participa y puede preguntar e interactuar con el usuario. De este tipo de observación se obtiene información de gran calidad.
El cuaderno de campo requiere menor esfuerzo dado que se entrega al usuario un cuaderno para rellenar cada vez que use el dispositivo y en él se puede observar no sólo el detalle de uso sino también las impresiones cualitativas del usuario.

Del trabajo de evaluación extraemos los objetivos de mejora y la definición para arrancar el nuevo ciclo de proyecto en espiral añadiendo nuevas funciones y opciones más complejas a las del ciclo anterior.

Al acabar cada ciclo es importante la difusión de los resultados para poder contrastar con otros proyectos, dada la escasez de experiencias en este terreno.

\section{Bibliografía}

Beyer, Hugh; Holtzblatt, Karen. Contextual design. Morgan Kaufmann, 1998. ISBN: 1558604111

Codina, Lluís. El llibre digital: una exploració sobre la informació electrònica y el futur de l'edició. Barcelona: Generalitat de Catalunya. Centre d'Investigació de la Comunicació, 1996. ISBN 84-393-3995-X

Codina, Lluís. Análisis y métodos en ciencias de la documentación. Consultado en 20-01-2008.

http://www.lluiscodina.com

Codina, Lluís. "El libro digital y el futuro del libro". En: El profesional de la información, 1998, v. 7, n. 1-2.

E-Ink. Electronic paper displays. Consultado en 20-02-2008.

http://www.eink.com/technology/

Eíto-Brun, Ricardo. "El camino hacia el libro electrónico". En: El profesional de la información, 2002, v. 11, n. 1, pp. 52-62.

Millán, José-Antonio. Libros y bitios. Consultado en 15-03-2008. http://jamillan.com/librosybitios/index.htm

Myebookdesign. Historia. Consultado en 21-03-2008 http://myebookdesign.com/sp/historia-libro-electronico.htm

Pawson, Dave. XSL-FO. Making XML look good in print. USA: O'Reilly \& Associates, Inc., 2002. ISBN 0596003552

Ramos, Manuel. Sobre los libros digitales. Consultado en 22-11-2007 http://www.sociedad20.com/20061022-sobre-los-libros-digitales.html

Rey, Ramón. Tinta electrónica. Consultado en 24-02-2008

http://www.referenta.com/tinta-electronica

Xerox Palo Alto Research Center. Electronic reusable paper. Consultado 20-03-2008

http://www2.parc.com/hsl/projects/gyricon/

Wikipedia. Spiral model. Consultado en 21-03-2008

http://en.wikipedia.org/wiki/Spiral_model

Wikipedia. Tinta electrónica. Consultado en 22-04-2008 http://es.wikipedia.org/wiki/Tinta_electr\%C3\%B3nica

XML Graphic Project. Consultado en 21-03-2008

http://xmlgraphics.apache.org/fop/

PabloLara-Navarra, plara@uoc.edu BegoñaGros-Salvat,bgros@uoc.edu MagíAlmirall, malmirall@uoc.edu Universitat Oberta de Catalunya, Avenida Tibidabo 47, 08035 Barcelona 